\title{
Can a community health worker administered postnatal checklist increase health-seeking behaviors and knowledge?: evidence from a randomized trial with a private maternity facility in Kiambu County, Kenya
}

Margaret McConnell ${ }^{1 *}$, Allison Ettenger ${ }^{2}$, Claire Watt Rothschild ${ }^{2}$, Faith Muigai ${ }^{2}$ and Jessica Cohen ${ }^{1}$

\begin{abstract}
Background: Since the 2009 WHO and UNICEF recommendation that women receive home-based postnatal care within the first three days after birth, a growing number of low-income countries have explored integrating postnatal home visit interventions into their maternal and newborn health strategies. This randomized trial evaluates a pilot program in which community health workers (CHWs) visit or call new mothers three days after delivery in peri-urban Kiambu County, Kenya.

Methods: Participants were individually randomized to one of three groups: 1) early postnatal care three days after delivery provided in-person with a CHW using a simple checklist, 2) care provided by phone with a CHW using the same checklist, or 3) a standard of care group. Surveys were conducted ten days and nine weeks postnatal to measure outcomes related to compliance with referrals, self-reported health problems for mother and baby, care-seeking behaviors, and postnatal knowledge and practices around the recognition of danger signs, feeding, nutrition, infant care and family planning.

Results: The home visit administration of the checklist increased the likelihood that women recognized postnatal problems for themselves and their babies and increased the likelihood that they sought care to address those problems identified for the child. In both the home visit and mobile phone implementation of the checklist, actions taken for postnatal problems happened earlier, particularly for infants. Knowledge was found to be high across all groups, with limited evidence that the checklist impacted knowledge and postnatal practices around the recognition of danger signs, feeding, nutrition, infant care and family planning.

Conclusion: We find evidence that CHW-administered postnatal checklists can lead to better recognition of postnatal problems and more timely care-seeking. Furthermore, our results suggest that CHWs can affordably deliver many of the benefits of postnatal checklists.
\end{abstract}

Trial registration: ClinicalTrials.gov NCT02104635; registered April 2, 2014.

Keywords: Postnatal care, Community health workers, Checklists, Mobile Health

\footnotetext{
* Correspondence: mmcconne@hsph.harvard.edu

'Harvard T. H. Chan School of Public Health, Building 1, Room 1217, 665

Huntington Ave, Boston, MA 02115, USA

Full list of author information is available at the end of the article
} 


\section{Background}

Despite overall gains in child survival, slower progress has been made in reducing neonatal and maternal mortality in developing countries [1]. Globally, a critical gap in the continuum of maternal and newborn health has been identified during the early postnatal period [2]. Recommendations from the World Health Organization indicate crucial moments when contact with skilled health providers could be instrumental in identifying and responding to the needs and complications of both mother and baby after childbirth: the first few hours after birth, between three and seven days, and at 6 weeks [3]. Many countries are now struggling to develop logistically feasible models of postnatal care delivery that address the numerous challenges women face in returning to facilities in the short interval after birth.

Neonatal and maternal mortality rates continue to be high in Kenya, with an estimated 488 maternal deaths per 100,000 live births and 22 neonatal deaths per 1,000 live births based on the most recently available Demographic and Health Survey [4, 5]. Further, maternal and neonatal mortality rates are higher among the urban poor [6]. A major driver of poor maternal and neonatal outcomes is low utilization of postnatal care. Only $47 \%$ of women in Kenya receive any postnatal care after delivery at all, and only $37 \%$ of women receive postnatal care from a medical professional such as doctor, nurse or midwife [4]. Poor women with lower levels of education are even less likely to receive this essential care [5]. Decisions about whether to seek care, delays in recognizing illness and reaching facilities, and low-quality care at health service points have been documented in neighboring Uganda as barriers to access to this essential care for newborns and their mothers [7].

A growing body of evidence demonstrates that home visits by community health workers (CHWs) during pregnancy and the postnatal period reduced rates of low birth weight, stillbirths and neonatal mortality [8-11]. Such home visits have been found to increase key practices in the promotion of neonatal health including early initiation and exclusive breastfeeding, thermal care, hand hygiene, umbilical cord care and increased care-seeking behavior for sick infants [7, 12-15]. Additionally in some settings CHWconducted home visits during the perinatal period demonstrated improvements in HIV-prevention strategies [8].

Despite the promise of early postnatal home visits, there is limited evidence on how to effectively implement postnatal care [16]. A Cochrane review of postnatal home visits examined 12 randomized trials both in high-resource and low-resource settings and found inconsistent results [17]. The review raises concerns that women who receive home visits may be less likely to seek care at facilities for their newborns if they feel they have already been sufficiently checked.

We conducted a randomized trial of a pilot program implemented three days after delivery in which a checklist was used by a community health worker to assess the health of the mother and newborn and targeted health education was offered. We compare the relative effectiveness of administering the checklist either inperson, as in more traditionally resource intensive $\mathrm{CHW}$ home visits, or by mobile phone, and compare both variations of the intervention to a standard of care group. The impact of the intervention with respect to identification of complications, care-seeking behaviors, health practices and knowledge was assessed.

\section{Methods}

Setting and study design

The intervention was implemented with Jacaranda Health, a private-sector social enterprise located in Kiambu County, Kenya providing high quality and lowcost maternal and newborn healthcare to low-income women in peri-urban areas of Nairobi. These regions are densely populated with characteristic challenges of poverty including poor access to water and sanitation, food insecurity and safety concerns $[6,18]$. Total fertility rates for women in urban poor regions are slightly higher than that of Nairobi (3.1 compared to 2.8) yet still lower than the national average of $4.6[4,6]$.

At Jacaranda Health's 10-inpatient bed hospital in a peri-urban setting just outside Nairobi, women were approached for recruitment and written informed consent after a normal delivery just prior to their discharge home. Women were eligible if they had a complicationfree delivery, their newborn experienced no visible complications, were over 18 years old, provided two phone numbers where they could be reached, and resided within $20 \mathrm{~km}$ of the Jacaranda Health facility. These eligibility criteria reflect Jacaranda Health's referral guidelines during the study period. During the study, Jacaranda Health had the capacity to conduct normal deliveries, provide basic emergency obstetric care and refer women who were at increased risk of a complication in their pregnancy to higher-level hospitals. Jacaranda's eligibility criteria were adapted from international guidelines including the World Health Organization and the U.K.'s National Institute for Clinical Excellence (NICE). The eligibility criteria excluded women with six or more past deliveries, advanced age (older than 35 years), abnormal values of ANC diagnostics (e.g. high blood pressure), history of medical complications, and history of obstetric complications.

The CHWs participating in our study were employed exclusively as Jacaranda staff and managed by a nurse midwife dedicated to outreach and community efforts. 
CHWs in our study were recruited from the larger area pool of CHWs who are recruited from barazas (local community meetings) and who receive foundational training in basic areas and are supervised by Community Health Extension Workers (CHEWs). The recruitment and training of these CHWs varies significantly in practice across Kenya [19].

The randomized trial took place between April 2014 and October 2014. Patients were individually randomized prior to enrollment using numeric patient identifiers assigned by Jacaranda Health. A unique identifier is given to each Jacaranda Health client seeking any service (including antenatal care, delivery, postnatal care, and child wellness care) during the client's first visit to Jacaranda. Randomization was conducted by assigning each of these unique identifiers to one of the three central treatment groups with equal probability: a $\mathrm{CHW}$ home visit three days after delivery, a phone call from a $\mathrm{CHW}$ three days after delivery, or a standard of care group that received a customer service phone call about their experience. ${ }^{1}$. At the start of study recruitment, all preexisting assigned Jacaranda identifiers were randomized. For clients who had attended antenatal care at Jacaranda, randomization was stratified using demographic and health variables collected in routine patient documentation. Identifiers were stratified by terciles of expected delivery date, distance from the client's home to the facility, and primiparity. Random assignments were also given to all future Jacaranda IDs not yet in use; these assignments were made to each of the three treatment groups with equal probability. Random assignment of patient identifiers was done using a randomization sequence generated by the principal investigators with STATA 11, (StataCorp, College Station, TX).

\section{Intervention design}

For women randomly assigned to the checklist groups, CHWs were trained to screen for maternal and newborn danger signs, to deliver targeted postnatal health education, and to refer mothers and their newborns to facilitybased care if necessary using a checklist to guide them through the process that was available in English and Kiswahili (Additional file 1). Community health worker training was conducted by the research program manager and a designated nurse for the program. Prior to independent administration of the intervention, CHWs shadowed other Jacaranda Health nurses conducting postnatal health education counseling after delivery and observed a nurse-conducted home visit.

CHWs contacted women either in their home or by mobile phone three days after delivery. The checklist was developed using international guidelines and academic publications and included components shown in Table 1 [12, 20, 21]. Newborn assessment characteristics
Table 1 Topics covered by day 3 postnatal checklist

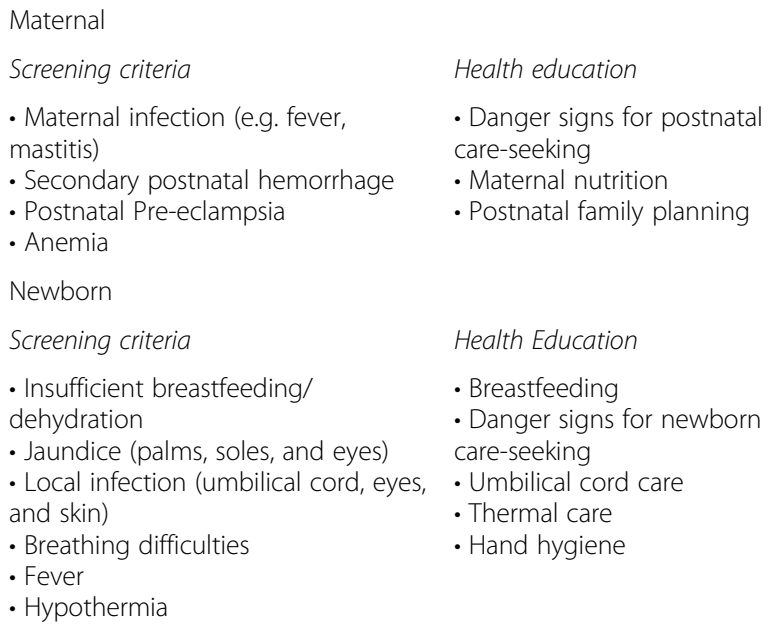

Newborn

Screening criteria Health Education

- Insufficient breastfeeding/ • Breastfeeding

dehydration - Danger signs for newborn

- Jaundice (palms, soles, and eyes) care-seeking

- Local infection (umbilical cord, eyes, - Umbilical cord care

and skin) - Thermal care

- Breathing difficulties • Hand hygiene

- Fever

- Hypothermia

such as poor feeding, fever, and jaundice also reflect the 2014 WHO guidelines. ${ }^{2}$ If a CHW detected a sign or symptom of maternal or neonatal illness through the assessment checklist on Day 3, the mother or child were referred to the nearest facility for curative care. ${ }^{3}$ The protocol specified that all complications, except for cracked nipples in isolation, were to be referred. For all referrals, a nurse conducted a phone follow-up the following day to ensure the woman and her child received appropriate care. Drafts of the checklist were locally pretested to ensure accuracy and comprehension among the communities and CHWs. Managers conducted regular audits of referral and checklist documentation to ensure appropriate completion and adherence to protocols.

CHWs completed a thorough four-day training to conduct screenings using the checklist and to counsel mothers and caregivers on essential postnatal health education. The training curriculum was adapted from international resources and modified to be relevant to specific programmatic contexts [20]. CHWs were evaluated through pre and post-test mechanisms to ensure thorough understanding and comprehension of essential information including: role-plays, observation of nurseconducted visit and calls and, upon concluding the training, were signed-off by a clinical staff that their performance met quality standards.

\section{Data collection}

Trained research assistants obtained written informed consent for participation in the study prior to discharge from the Jacaranda Health hospital in Ruiru. Recruitment and survey administration occurred on a rolling basis throughout the study period based on the date that women delivered at the Jacaranda Health hospitals.

Estimates of program impact were analyzed using administrative, programmatic, and survey data. Administrative 
data is routinely collected for all Jacaranda Health clients and uploaded into their medical records; these data include information on patient history and health services received, as well as demographic data. Electronic medical records data were available for all enrolled participants. The second data source was programmatic data collected by the $\mathrm{CHW}$ during administration of the checklist. The final source of data was follow-up participant surveys, administered by phone by trained research assistants at ten days and nine weeks after delivery.

All participants were contacted at each data collection time point, regardless of whether they were previously reached (including those who were never reached by either a home visit or phone call on the third day after giving birth). Inability to reach participants for both the day 3 interventions and the follow-up phone surveys was assessed based on the following protocols: For the day 3 interventions, participants assigned to receive a customer service call (standard of care arm) or phone administration of the checklist were contacted up to four times by phone on the scheduled day (using all phone contact information provided by the participant at enrollment). The participant was considered unreachable if none of the four calls were answered. For the home visit arm, the CHW contacted the participant by phone on the morning of her scheduled visit to confirm the location of the residence, and subsequently knocked on the door or otherwise introduced herself at the home. If the participant was not reachable by phone while the $\mathrm{CHW}$ was in the field that day or was not available at the home for the entire day, she was considered unable to be reached by the checklist treatment.

Regardless of whether the participants were reached on day 3 , all were scheduled for follow-up mobile phone surveys at ten days and nine weeks after delivery. Attempts were made to contact participants for the day 10 follow-up survey beginning on day 10 after delivery, with daily contact attempts made for the subsequent seven days after the scheduled date. If the participant was unreachable or unable to complete the phone survey during this time, she was considered unreachable. Likewise, attempts were made to contact participants for the nine week survey beginning on the scheduled date, with daily contact attempts made for a subsequent 14 days.

\section{Outcomes}

Postnatal maternal and newborn outcomes are captured at three time periods - three days, ten days, and nine weeks post-delivery. Day 3 outcomes were collected during administration of the checklist by the CHW and include maternal or newborn complications detected and referrals made for complications. These outcomes were collected in both treatment arms but not the standard of care arm. Day 10 and 9 week survey outcome measures include care-seeking behaviors for mother and newborns and both knowledge and practice of infant care, nutrition, feeding and recognition of danger signs. Table 2 provides definitions of all outcome measures.

In order to avoid concerns about multiple outcome testing, levels of postnatal health knowledge were assessed by constructing summative indices of the number of items that participants were able to name in response to knowledge questions as suggested by O'Brien [22] and Kling and Liebman (2004) [23]. Knowledge outcomes were also converted into binary variables, indicating whether the participant was able to name a specified number of knowledge items.

\section{Ethical approval trial registration}

This study was approved by Institutional Review Boards at Harvard School of Public Health and the Ethical and Scientific Review Committee of African Medical Research Foundation (AMREF) in Nairobi, Kenya. The study design was registered at clinicaltrials.gov with identification number NCT02104635.

\section{Statistical power}

Because of the small sample of this study, our study is powered to see only large changes in the primary careseeking outcomes. With our original registered sample size of 109 individuals, we had $80 \%$ power to detect a change of 30 percentage points in maternal care-seeking from a base of $8 \%$ of women seeking care, using a twosided test comparing any two treatment groups and a $5 \%$ significance level threshold. Because child care seeking levels are so high, even with the standard of care (over $95 \%$ of the sample seeks some care for their newborn prior to the 10 day survey), we were not powered on this outcome. Because of loss to follow-up our actual sample size for the survey conducted 10 days after delivery was 83 . With our final sample, we have $80 \%$ power to detect a change of 37 percentage points in maternal care-seeking compared to the standard of care, using a two-sided pairwise test and a $5 \%$ significance level. Power calculations were conducted using STATA, version 12.1.

\section{Data analysis}

We test for differences in outcomes across study arms on an intention-to-treat basis, with the treatment arms defined as participants' randomized treatment assignment and the sample including all respondents where data is available (not just those reached by a day 3 intervention). We also provide Additional file 2: Tables A1A3, which recreate all regression analyses restricted to the subset of participants who were successfully reached for the day 3 intervention. 
Table 2 Definition of primary outcomes

Outcome measure

Maternal complication detected and referred

Newborn complication detected and referred

\section{Outcomes reported at 10 days postpartum}

Maternal referral acted upon

Newborn referral made and acted upon

Facility-based maternal postpartum care sought

Days postpartum maternal care sought, among maternal care-seekers

Facility-based newborn postpartum care sought

Days postpartum newborn care sought, among maternal care-seekers

Any maternal health problem reported

Maternal problem reported and action taken

Any newborn health problem reported

Newborn problem reported and action taken

Number of maternal danger signs named

Can name 3 or more maternal danger signs

Number of newborn danger signs named

Can name 3 or more newborn danger signs

Can name 2 or more hand washing best practices

Can name 2 or more cord care practices

Can name 3 or more newborn thermal care practices

Can name 3 or more sources of dietary protein

Applied water or nothing to umbilical stump
Definition

CHWs documented any observed or reported maternal complications detected through administration of the checklist; data collected for study participants assigned to CHW treatment by phone or home visit

CHWs documented any observed or reported complications detected through administration of the checklist; data collected for study participants assigned to CHW treatment by phone or home visit

Binary variable indicating CHW referral was made (from programmatic data) and respondent reporting seeking facility-based care based on referral advice

Binary variable indicating CHW referral was made (from programmatic data) and respondent reporting seeking facility-based care based on referral advice

Binary variable indicating whether respondent reported going to a health facility for her health since delivery

Continuous variable indicating days after delivery maternal care was sought, for those who report seeking facility-based maternal care

Binary variable indicating whether respondent reported bringing her baby for a child wellness clinic visit

Continuous variable indicating days after delivery newborn wellness care was sought, for those who report seeking facility-based newborn wellness care

Binary variable indicating respondent reported yes to the question, "Are you having any problems with your health, related to your delivery, since you delivered?"

Binary variable indicating respondent reported yes to the question, "Did you do anything as a response to this problem?", administered to respondents who reported a newborn health problem.

Binary variable indicating respondent reported yes to the question, "Is your baby having any problems with his/her health since you delivered?"

Binary variable indicating respondent reported yes to the question, "Did you do anything as a response to this problem?", administered to respondents who reported a newborn health problem.

Accurate identification of maternal danger signs defined as ability to name any of the following 11 predefined signs: fever/chills, foul-smelling vaginal discharge, convulsions/loss of consciousness, heavy vaginal bleeding (defined as soaking through a pad every hour), severe headaches, dizziness or faintness, visual disturbance (blurry vision or unusual difficulty seeing), increased cramping or abdominal pain, increased perineal pain, swelling, redness or discharge, difficulty passing urine, difficulty breathing, breast redness or hot to the touch, and pain or lump in the breast.

Binary variable coded for continuous outcome variable, indicating whether 3 or more maternal dangers signs were named

Accurate identification of infant danger signs defined as ability to name any of the following eight signs: fever, jaundice, poor feeding, lethargic/unresponsive, umbilical cord redness or discharge, convulsions, abnormal breathing (including panting, fast breathing, grunting, or nasal flaring, and pus from eyes.

Binary variable coded for continuous outcome variable, indicating whether 3 or more newborn dangers signs were named

Accurate identification of hand washing best practices defined as ability to name any of the following times that are particularly important for a caregiver to wash hands: after using the toilet, before touching/holding baby, after washing or touching nappies

Accurate identification of sources of cord care practices defined as ability to name any of the following best practices to prevent cord infection: keep cord clean and dry, do not apply anything to stump, keep cord outside nappy/diaper

Accurate identification of sources of dietary protein defined as ability to name any of the following best practices to ensure baby stays warm: keep room where newborn stays warm, dress newborn in several layers of clothes, bathe baby quickly in cold weather using warm water and dry and dress baby quickly, keep newborn's head covered, practice skin to skin contact

Accurate identification of sources of dietary protein defined as ability to name any of the following: beans, lentils, meat, eggs, chicken, fish, milk

Binary variable indicating nothing or water applied to umbilical stump. The checklist and data collection defined best practices in umbilical cord care according to Jacaranda Health's clinical practice of dry cord care at the time of data collection. While Kenya introduced a change in its 
Table 2 Definition of primary outcomes (Continued)

\begin{tabular}{|c|c|}
\hline & $\begin{array}{l}\text { newborn care clinical guidelines from dry care to use of topical antiseptic in November } 2013 \text {, the } \\
\text { World Health Organization recommends both dry cord care and topical antiseptic as best practice } \\
{[33,34] \text {. }}\end{array}$ \\
\hline Appropriate newborn thermal care practiced & $\begin{array}{l}\text { Binary variable indicating respondent mentions trying to keep baby warm when asked how she } \\
\text { bathes her baby }\end{array}$ \\
\hline Exclusive breastfeeding & Binary variable indicating only newborn consumed only breastmilk since birth \\
\hline Breastfed 3 or more times in past 8 hours & $\begin{array}{l}\text { Binary variable indicating respondent reports feeding baby } 3 \text { or more times in } 8 \text { hours prior to the } \\
\text { survey }\end{array}$ \\
\hline \multicolumn{2}{|l|}{ Outcomes reported at 9 weeks postpartum } \\
\hline $\begin{array}{l}\text { At least one dose of polio and pentavalent } \\
\text { vaccines }\end{array}$ & $\begin{array}{l}\text { Binary variable indicating that respondent reports infant has received at one or more doses of polio } \\
\text { vaccine and one or more doses of pentavalent vaccine when asked which vaccinations the infant } \\
\text { has received since delivery }\end{array}$ \\
\hline Use of family planning method & Binary variable indicating respondent reports current use of any family planning method \\
\hline Exclusive breastfeeding & Binary variable indicating only newborn consumed only breastmilk since birth \\
\hline Breastfed 3 or more times in past 8 hours & $\begin{array}{l}\text { Binary variable indicating respondent reports feeding baby } 3 \text { or more times in } 8 \text { hours prior to the } \\
\text { survey }\end{array}$ \\
\hline \multicolumn{2}{|l|}{ Index measures } \\
\hline $\begin{array}{l}\text { Index of health knowledge at } 10 \text { days post- } \\
\text { delivery }\end{array}$ & $\begin{array}{l}\text { Summative index with a maximum of } 6 \text { points and a minimum of } 0 \text { points, where each point } \\
\text { represents knowledge of the following } 6 \text { postnatal health topics: } 1 \text { ) ability to name } 3 \text { or more } \\
\text { maternal danger signs; } 2 \text { ) ability to name } 3 \text { or more infant danger signs; } 3 \text { ) ability to name } 2 \text { or } \\
\text { more hand washing best practices; } 4 \text { ) ability to name } 2 \text { or more; 5) ability to name } 3 \text { or more } \\
\text { recognized newborn thermal care practices; } 6 \text { ) and ability to name } 3 \text { or more sources of maternal } \\
\text { dietary protein }\end{array}$ \\
\hline $\begin{array}{l}\text { Index of health practices at } 10 \text { days and } \\
9 \text { weeks post-delivery }\end{array}$ & $\begin{array}{l}\text { Summative index with a maximum of } 8 \text { points and a minimum of } 0 \text { points, where } 4 \text { points } \\
\text { represent } 4 \text { key health practices reported at } 10 \text { day (exclusive breastfeeding, breastfed } 3 \text { or more } \\
\text { times in the past } 8 \text { hours, appropriate newborn thermal care practices, and water or nothing applied } \\
\text { to umbilical cord stump) and } 4 \text { points represent } 4 \text { key health practices reported at } 9 \text { weeks } \\
\text { (exclusive breastfeeding, breastfed } 3 \text { or more times in the past } 8 \text { hours, use of postnatal } \\
\text { contraception, infant has received at least one dose of polio and pentavalent vaccines). }\end{array}$ \\
\hline
\end{tabular}

Program impacts were estimated using logistic regression for binary outcomes and ordinary least squares (OLS) regression for continuous outcomes. OLS and logistic estimates of program impact are presented both in unadjusted and adjusted models. Outcomes of interest are regressed on separate binary variables for assignment to one of the treatment arms. Adjusted estimates include controls for female participant's age, marital status, employment status in the past 12 months, attendance at one or more antenatal care visits at a Jacaranda Health facility, and a binary indicator for whether the participant was enrolled in a postnatal contraceptive subsidy program as part of a concurrent randomized controlled trial. All analyses were performed using Stata software, version 12.1 (StataCorp, College Station, TX).

Participant flow through enrollment, administration of the day 3 interventions, and follow-up surveying are presented in Fig. 1. Programmatic data were collected for the 77 women who were successfully reached at day 3 by the assigned intervention. The rate of reaching individuals at day 3 for the intervention was higher in the checklist call group (76 \%) compared to the checklist home visit group (59\%). This difference may be due to difficulties finding participants either because they are traveling or because their households were difficult to locate. Participant survey data were collected for 83 participants at ten days post-delivery (24 in the standard of care arm, 32 in the phone-administered checklist arm, and 27 in the home visit-administered checklist arm). Participant surveys were collected for 59 participants at nine weeks after delivery (17 in the standard of care arm, 23 in the phone arm, and 19 in the home visit arm). Four participants were unable to be contacted at any data collection point after enrollment, due to errors in their contact details.

\section{Results}

Demographic and household characteristics of the sample included as controls in adjusted models are presented by treatment arm in Table 3. Participants are on average 26-27 years old, with less than $1 \%$ of the total sample older than 35 . The majority of participants are married, have received at least some secondary schooling, and have had previous pregnancies. Most of the differences in characteristics across arms are small and not statistically significant, except for a somewhat lower rate of marriage in the call arm (85\% (35/41) vs. $97 \%(30 /$ 31 ) and $97 \%(30 / 31)$ in the standard of care and home visit arms, respectively) and a lower employment rate in the visit $\operatorname{arm}(41 \%(11 / 27)$ vs. $66 \%(21 / 32)$ to $70 \%(16 /$ 


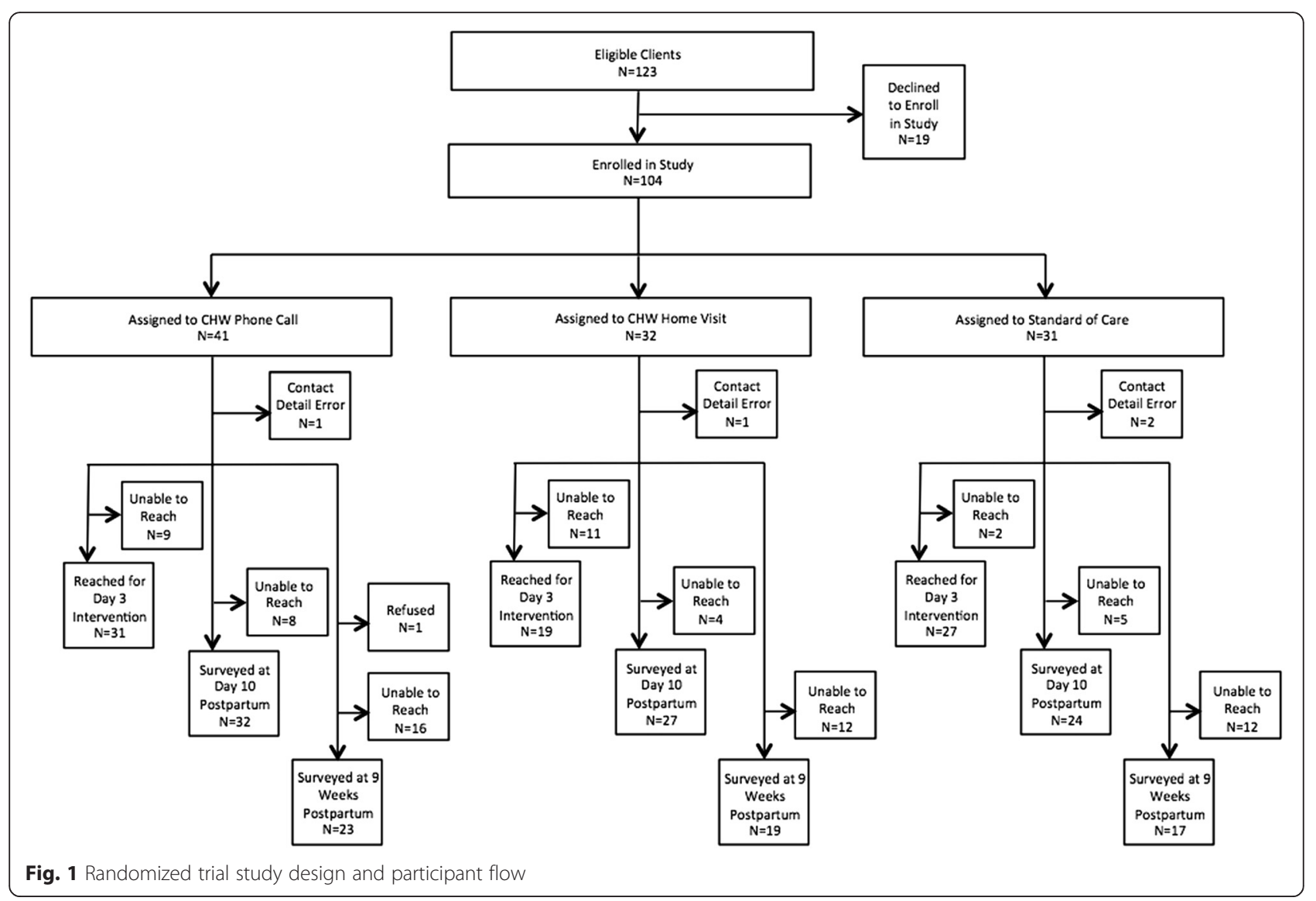

23 in the other arms). While the difference in employment status in the visit arm is substantial, it does not seem to reflect overall imbalance in socioeconomic status across arms, as households in the visit arm are not significantly less educated or less likely to have improved toilets or water supplies. A subsample of our participants was concurrently enrolled in a study that provided a voucher for free postpartum family planning. Since assignment to the voucher arm was higher in the CHW call group arm we include this voucher assignment as a control in all adjusted models.

\section{Postnatal complication detection and referral at 3 days after delivery}

At the day 3 visit, CHWs documented any observed or reported maternal and newborn complications detected through administration of the checklist. Complications were detected and referrals made for $10 \%(4 / 41)$ and $9 \%(3 / 32)$ of the phone call and home visit arms, respectively, with no significant difference in referral percentage by arm (unadjusted $95 \% \mathrm{CI}:-0.14-0.14 ; \mathrm{p}=$ 0.957). All complications detected related to newborn danger signs, with no maternal complications detected in any arm. As reported by clients at day 10 survey follow-up, $4 \%(1 / 23)$ and $11 \%(2 / 18)$ of participants both had a referral and acted upon it in the phone call and home visit arms, respectively. No difference was found in referrals acted upon in the home visit relative to the phone call arms (unadjusted $95 \% \mathrm{CI}$ : $-0.11-0.25$; $p=0.445)$.

\section{Care-seeking practices at $\mathbf{1 0}$ days after delivery}

Table 4 presents participant-reported health-seeking practices at the ten-day postnatal follow-up survey. Panel A presents maternal-focused care sought for any reason at a health facility, including routine postnatal care. Relative to $8 \%(2 / 24)$ of women in the standard of care arm who sought maternal care, participants in the phone call treatment arm were 1.6 times more likely to have sought maternal care by day 10 (unadjusted $95 \%$ CI: $0.26-9.49 ; p=0.622$ ), while participants in the home visit arm were three times as likely (unadjusted $95 \%$ CI: 0.56-17.5; $p=$ 0.192). Differences between the standard of care arm and each treatment arm are not statistically significant in the crude model; facility-based care seeking is higher in the home visit arm relative to the standard of care arm in the adjusted model (adjusted $95 \%$ CI: $0.77-71.5 ; p=0.082$ ), though the result is not quite significant at the $5 \%$ level. Relative to 
Table 3 Descriptive statistics of participant demographics and primary outcomes

Panel A. Participant demographic

and household characteristics

Standard of care CHW call CHW visit $\quad$-values

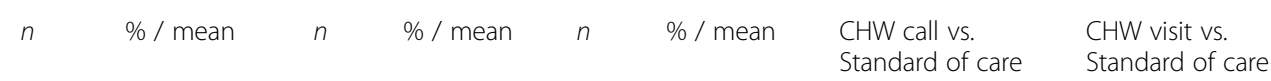

Women

Age

$31 \quad 26.7$

$40 \quad 25.6$

$31 \quad 25.9$

0.23

0.38

Education

$\begin{array}{lllllll}\text { Primary } & 4 & 14 \% & 8 & 21 \% & 4 & 15 \% \\ \text { Secondary } & 15 & 52 \% & 18 & 47 \% & 16 & 59 \% \\ \text { Post-Secondary } & 10 & 35 \% & 12 & 32 \% & 7 & 26 \% \\ \text { n } & 19 & & 38 & & 27 & \end{array}$

Marital Status

\section{Married \\ Single}

Parity

Primiparous

Multiparous

Employed in

Past 12 Months

n

Assigned to postnatal

contraception subsidy

treatment through

concurrent randomized trial

Husband

Husband Education

$\begin{array}{lllllll}\text { Primary } & 0 & 0 \% & 4 & 14 \% & 2 & 8 \% \\ \text { Secondary } & 15 & 65 \% & 13 & 45 \% & 10 & 39 \% \\ \text { Post-Secondary } & 8 & 35 \% & 12 & 41 \% & 14 & 54 \% \\ \mathrm{n} & 23 & & 29 & & 26 & \end{array}$

Household

Improved toilet facility

Toilet shared among

more than one house

n

Improved drinking

water source

Panel B. Primary

outcomes

\begin{tabular}{|c|c|c|c|c|c|c|c|}
\hline 24 & $92 \%$ & 34 & $97 \%$ & 24 & $86 \%$ & 0.43 & 0.45 \\
\hline 26 & & \multicolumn{2}{|l|}{35} & \multicolumn{4}{|l|}{28} \\
\hline 9 & $39 \%$ & 15 & $47 \%$ & 10 & $39 \%$ & 0.58 & 0.96 \\
\hline 23 & & \multicolumn{2}{|c|}{32} & \multicolumn{4}{|l|}{26} \\
\hline 8 & $90 \%$ & 27 & $96 \%$ & 23 & $92 \%$ & 0.41 & 0.82 \\
\hline مح & & \multicolumn{2}{|c|}{28} & \multicolumn{4}{|l|}{25} \\
\hline \multicolumn{2}{|c|}{ Standard of Care } & \multicolumn{2}{|c|}{ CHW call } & \multicolumn{2}{|c|}{ CHW visit } & p-values & \\
\hline & $\%$ / mean & $\mathrm{n}$ & $\%$ / mean & $n$ & $\%$ / mean & $\begin{array}{l}\text { CHW call vs. } \\
\text { Standard of care }\end{array}$ & $\begin{array}{l}\text { CHW visit vs. } \\
\text { Standard of care }\end{array}$ \\
\hline
\end{tabular}


Table 3 Descriptive statistics of participant demographics and primary outcomes (Continued)

Complications detected and referred, collected

through programmatic

CHW data

Maternal or newborn

complications detected

4

41

Postpartum care-seeking practices for mother and newborn, reported during day 10 survey

Referrals made and acted upon

Facility-based maternal postpartum care sought

Days postpartum maternal care sought, among maternal care-seekers

Facility-based newborn postpartum care sought

Days postpartum newborn care sought, among newborn care-seekers

Postnatal health problems and responses at day

10 post-delivery

Any maternal health problem reported

Maternal problem reported and action taken

Any newborn health problem reported

Newborn problem reported and action taken

$$
2
$$$$
8 \%
$$$$
24
$$$$
2
$$$$
8.5
$$

$96 \%$

24

23

5.9

1

$4 \%$

23

4

$13 \%$

32

4

7.5

30

$94 \%$

32

30

3.8

$3 \quad 13 \%$

24

$2 \quad 8 \%$

24

$5 \quad 21 \%$

24

24

Self-reported knowledge of postnatal danger signs and health practices at day 10

Number of maternal danger signs named

Can name 3 or more maternal danger signs

Number of newborn danger signs named

Can name 3 or more newborn danger signs
24

$19 \quad 79 \%$

24

24

19
$10 \%$

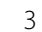

$9 \%$

32 
Table 3 Descriptive statistics of participant demographics and primary outcomes (Continued)

\begin{tabular}{|c|c|c|c|c|c|c|c|c|}
\hline$n$ & 24 & & 32 & & 27 & & & \\
\hline $\begin{array}{l}\text { Can name } 2 \text { or more } \\
\text { hand washing best practices }\end{array}$ & 10 & $42 \%$ & 19 & $59 \%$ & 10 & $37 \%$ & 0.20 & 0.74 \\
\hline$n$ & 24 & & 32 & & 27 & & & \\
\hline $\begin{array}{l}\text { Can name } 2 \text { or more } \\
\text { cord care practices }\end{array}$ & 7 & $29 \%$ & 13 & $41 \%$ & 5 & $19 \%$ & 0.38 & 0.39 \\
\hline$n$ & 24 & & 32 & & 27 & & & \\
\hline $\begin{array}{l}\text { Can name } 3 \text { or more } \\
\text { newborn thermal } \\
\text { care practices }\end{array}$ & 7 & $29 \%$ & 11 & $34 \%$ & 8 & $30 \%$ & 0.68 & 0.97 \\
\hline$n$ & 24 & & 32 & & 27 & & & \\
\hline $\begin{array}{l}\text { Can name } 3 \text { or more } \\
\text { sources of dietary protein }\end{array}$ & 7 & $29 \%$ & 12 & $38 \%$ & 11 & $41 \%$ & 0.52 & 0.40 \\
\hline$n$ & 24 & & 32 & & 27 & & & \\
\hline \multicolumn{9}{|l|}{$\begin{array}{l}\text { Self-reported postnatal } \\
\text { health practices at day } 10\end{array}$} \\
\hline $\begin{array}{l}\text { Applied water or } \\
\text { nothing to umbilical stump }\end{array}$ & 17 & $71 \%$ & 26 & $81 \%$ & 23 & $85 \%$ & 0.38 & 0.23 \\
\hline$n$ & 24 & & 32 & & 27 & & & \\
\hline $\begin{array}{l}\text { Appropriate newborn } \\
\text { thermal care practiced }\end{array}$ & 17 & $71 \%$ & 26 & $81 \%$ & 23 & $85 \%$ & 0.84 & 0.93 \\
\hline $\mathrm{n}$ & 24 & & 32 & & 27 & & & \\
\hline Exclusive breastfeeding & 24 & $100 \%$ & 32 & $100 \%$ & 27 & $100 \%$ & - & - \\
\hline $\mathrm{n}$ & 24 & & 32 & & 27 & & & \\
\hline $\begin{array}{l}\text { Breastfed } 3 \text { or more } \\
\text { times in past } 8 \text { hours }\end{array}$ & 23 & $96 \%$ & 27 & $84 \%$ & 25 & $93 \%$ & 0.14 & 0.63 \\
\hline$n$ & 24 & & 32 & & 27 & & & \\
\hline \multicolumn{9}{|l|}{$\begin{array}{l}\text { Self-reported postnatal } \\
\text { health practices } \\
\text { at } 9 \text { weeks post-delivery }\end{array}$} \\
\hline $\begin{array}{l}\text { At least } 1 \text { dose of polio } \\
\text { and pentavalent vaccines }\end{array}$ & 15 & $88 \%$ & 22 & $96 \%$ & 18 & $95 \%$ & 0.43 & 0.50 \\
\hline$n$ & 17 & & 23 & & 19 & & & \\
\hline $\begin{array}{l}\text { Use of family planning } \\
\text { method }\end{array}$ & 5 & $29 \%$ & 14 & $61 \%$ & 8 & $42 \%$ & 0.05 & 0.44 \\
\hline$n$ & 17 & & 23 & & 19 & & & \\
\hline Exclusive breastfeeding & 17 & $100 \%$ & 21 & $91 \%$ & 19 & $100 \%$ & 0.16 & - \\
\hline$n$ & 17 & & 23 & & 19 & & & \\
\hline $\begin{array}{l}\text { Breastfed } 3 \text { or more times } \\
\text { in past } 8 \text { hours }\end{array}$ & 15 & $88 \%$ & 22 & $96 \%$ & 18 & $95 \%$ & 0.43 & 0.50 \\
\hline$n$ & 17 & & 23 & & 19 & & & \\
\hline \multicolumn{9}{|l|}{$\begin{array}{l}\text { Indices of postnatal } \\
\text { health knowledge } \\
\text { and practices at } 10 \text { days } \\
\text { and } 9 \text { weeks post-delivery }\end{array}$} \\
\hline $\begin{array}{l}\text { Index of health knowledge } \\
\text { at } 10 \text { days post-delivery }\end{array}$ & 24 & 3.17 & 32 & 3.56 & 27 & 3.15 & 0.37 & 0.96 \\
\hline $\begin{array}{l}\text { Index of health practices } \\
\text { at } 10 \text { days and } 9 \text { weeks } \\
\text { post-delivery }\end{array}$ & 15 & 6.6 & 20 & 7.1 & 17 & 7.2 & 0.16 & 0.06 \\
\hline
\end{tabular}


Table 4 Postnatal care-seeking practices for mother and newborn reported during day 10 survey

Panel A. Maternal postnatal care seeking

$$
\text { (1) }
$$

Facility-based maternal postnatal care sought

Facility-based materna postnatal care sought

(3)

Days postnatal maternal care-seekers

Unadjusted Adjusted Unadjusted

Estimation Type

Logistic

Logistic

1.57

$(0.26-9.49)$

1.63

$(0.23-11.8)$

$7.44^{*}$

CHW home visit

3.14

$(0.56-17.5)$

0.08

0.330

$(0.77-71.5)$

Standard of care arm mean

$p$-value for test of call = visit

0.08

0.105

0.161

of call and visit

Controls?

R-squared

Observations

N

Y

OLS

$-1$

$(-4.52-2.52)$

$-2.67^{*}$

$(-5.66-0.33)$

8.5

0.417

0.173

N

0.159

12

83

79

(2)

(3)

(1)

Facility-based newborn postnatal care sought ${ }^{\ddagger \neq}$

Facility-based newborn postnatal care sought ${ }^{\ddagger}$

Days postnatal newborn care sought, among newborn care-seekers

Unadjusted Adjusted Unadjusted

Estimation Type

CHW phone call

Logistic

Logistic

0.65

(0.055 - 7.76)

1.13

0.47

(0.054 - 4.10)

0.51

(0.066 - 19.4)

$(0.022-11.8)$

0.96

0.96

0.942

0.791

OLS

$-2.04 * *$

$(-3.65--0.42)$

$-1.79 * *$

$(-3.45--0.14)$

5.86

0.714

0.041

N

0.098

79
(4)

Days postnatal materna care-seekers

Adjusted

OLS

$-1.05$

$(-10.3-8.23)$

$-2.27$

$(-14.2-9.65)$

8.5

0.769

0.833

Y

0.438

10

(4)

Days postnatal newborn care sought, among newborn care-seekers

Adjusted

OLS

$-2.27 * * *$

$(-3.89--0.64)$

$-1.78^{*}$

$(-3.69-0.13)$

5.86

0.537

0.025

Y

0.195

75

* The binary covariate for receipt of a postnatal contraception subsidy is dropped due to collinearity.

\#\# The reduced number of observations in Panel B (columns 1 and 2 ) is due to the low variation in the outcomes across treatment arms, with $94.3 \%$ of the total sample reporting having sought newborn care by the day 10 survey. In model (1), the coefficient on the CHW phone call intervention cannot be estimated due to this lack of variation in the outcome relative to the standard of care arm. Likewise, no adjusted model is presented in column (2) due to the reduced number of observations when covariates are included in the model. Notes: Odds ratios generated via logistic regression (models 1,2 ) are presented with $95 \%$ confidence intervals constructed with robust standard errors. Maternal care seeking (panel A, columns 1 and 2) is defined as a binary variable, taking on the value of 1 if the woman reports seeking facility-based maternal-related care. Infant care seeking (panel B, columns 1 and 2 ) is defined as a binary variable, taking on the value of 1 if the woman reports attending a child wellness visit. OLS coefficients are presented for models 3 and 4 . Days postnatal maternal care sought (panel A, columns 3 and 4) is defined as a continuous variable of the number of days that maternal care was sought after the date of delivery. Days postnatal infant care sought (panel B, columns 3 and 4) is likewise defined as a continuous variable of the number of days that care for the infant was sought after the date of delivery. For all adjusted regressions (columns 2 and 4), individual level covariates include the female participant's age (coded as an ordinal variable with values 18-25, 26-30, 31-35, or 36-40 years old), marital status (defined as a binary single or married), a binary variable indicating whether the female respondent was employed at any time in the past 12 months, a binary variable indicating whether the participant was enrolled in a concurrent randomized trial and received a voucher (cost subsidy) for postnatal family planning services, and a binary variable indicating whether the participant attended one or more antenatal care visits at a Jacaranda Health facility 
maternal care seeking, facility-based infant-related care seeking was very high across arms (Panel B): $96 \%(23 / 24)$ of standard of care arm participants reported seeking infant-related care (including immunizations and child wellness visits) by ten days after delivery, as compared to $94 \%(30 / 32)$ and $96 \%$ (26/ 27 ) of the phone and visit arms, respectively.

We find evidence of program impacts on the timing of care-seeking for both mother and infant. Among participants who reported seeking facility-based maternal care, participants assigned to the home visit arm sought care on average 2.7 days sooner than the standard of care arm (unadjusted $95 \% \mathrm{CI}:-5.66-0.33$; $p=0.075)$, though this result is not quite statistically significant at the $5 \%$ level. Statistically significant differences are found in the timing of infant-related care seeking: among participants who sought facility-based infant-related care, assignment to the phone call and home visit resulted in infant care-seeking $2.0(p=0.014)$ and 1.8 days $(p=0.034)$ earlier than the standard of care.

In Table 5, we present self-reported maternal (Panel A) and newborn (Panel B) health problems and responses taken. $13 \%(3 / 24)$ of women assigned to the standard of care arm reported experiencing a maternal health problem, and $8 \%(2 / 24)$ both experiencing a problem and reporting taking action to address this health problem. Assignment to the home visit is estimated to increase the likelihood of reporting a maternal problem by 3.5 times (unadjusted $95 \%$ CI: $0.81-15.1 ; p=0.092$ ) to 5.6 times (adjusted $95 \%$ CI: $0.88-35.4 ; p=0.068$ ) relative to the standard of care group, depending on model specification, though these effects do not meet a standard of $5 \%$ significance level. The odds of reporting a maternal health problem in the call arm were not significantly different from the standard of care group and were significantly higher in the home visit arm than in the call arm (unadjusted $95 \%$ CI: 1.43-39.20; $p=.017$ ). While the odds of reporting a maternal problem and taking action were higher in the home visit arm than in the standard of care arm, these differences were not statistically significant.

We find that $21 \%(5 / 24)$ of women assigned to the standard of care arm reported newborn health problems, with $13 \%(3 / 24)$ reporting both noticing and responding to a newborn health problem. Women assigned to the home visit were 3.5 times (unadjusted $95 \%$ CI: 1.01-12.3; $p=$ 0.048 ) to 3.8 times (adjusted $95 \%$ CI: 0.95-15.1; $p=0.060$ ) more likely to report newborn health problems. Women in the home visit arm were 2.95 (unadjusted $95 \% \mathrm{CI}$ : 0.68$12.9 ; p=0.151$ ) more likely to both report and take action on a newborn problem than in the standard of care group in the unadjusted model, and 3.94 (adjusted 95 \% CI: 0.8019.4; $p=0.092$ ) times more likely to do so in the adjusted model, although estimates are not statistically significant at the $5 \%$ level.

\section{Postnatal knowledge at $\mathbf{1 0}$ days after delivery}

Figure 2 presents the percentage of women in each treatment arm with knowledge of six key postnatal health knowledge items. $85 \%(23 / 27)$ of women assigned the visit arm were able to name three or more maternal danger signs, as well as three or more infant danger signs. $81 \%(26 / 32)$ of the phone call arm was able to name three or more maternal danger signs, with $78 \%(25 / 32)$ able to name three or more infant danger signs. $79 \%(19 / 24)$ and $67 \%(16 / 24)$ of women in the standard of care arm able to name three or more maternal and infant danger signs, respectively.

\section{Health practices, reported at 10 days and 9 weeks postnatal}

Postnatal health practices at 10 days after delivery are presented in Panel A of Fig. 3. We find 100 \% (83/83) of the surveyed sample reporting exclusive breastfeeding, $96 \%(80 / 83)$ reporting practicing appropriate newborn thermal care practices while bathing, and $90 \%(75 / 83)$ reporting frequent breastfeeding (defined as breastfeeding 3 or more times in the previous 8 hours). We find the highest level of variation in short-term postnatal health practices in umbilical cord care, with $85 \%$ (23/ 27 ) of the home visit arm and $81 \%(26 / 32)$ phone call arm reporting applying water or nothing to the stump (as recommended), compared to $71 \%(17 / 24)$ of the standard of care arm. We find no statistically significant differences in short-term postnatal health practices by treatment assignment.

Self-reported health practices at nine weeks after delivery are presented in Panel B of Fig. 3. As with the short-term health practices, we find very high reported compliance with recommended health practices at nine weeks post-delivery. We find no significant differences in exclusive breastfeeding, frequency of breastfeeding, or receipt of routine infant immunizations by treatment arm. We find an increased likelihood of family planning use by nine weeks after delivery in both the phone call and home visit arms relative to the standard of care, with those assigned to the phone call having between 3.7 times (unadjusted $95 \%$ CI: $0.97-14.4 ; p=0.06$.) and 3.1 times increased odds (adjusted 95 \% CI: 0.51-18.7; $p$ $=0.22$ ) and those assigned to the home visit arm having between 1.7 times (unadjusted $95 \%$ CI: 0.43-7.1; $p=0.43$ ) and 2.2 times increased odds (adjusted $95 \%$ CI: $0.35-13.4 ; p=0.41)$ of reporting family planning, although these results are not statistically significant. ${ }^{4}$

In Table 6, we present regression estimates using ordinary least squares where the outcomes are summative indices of postnatal health knowledge at day 10 postdelivery (unadjusted model in column 1; adjusted model in column 2) and short- and long-term postnatal health 
Table 5 Postnatal health problems and responses as reported during day 10 survey

\begin{tabular}{|c|c|c|c|c|}
\hline \multicolumn{5}{|c|}{ Panel A. Maternal health problem reporting and responses } \\
\hline & $(1)$ & (2) & (3) & (4) \\
\hline & $\begin{array}{l}\text { Any maternal health } \\
\text { problem reported }\end{array}$ & $\begin{array}{l}\text { Any maternal health } \\
\text { problem reported }\end{array}$ & $\begin{array}{l}\text { Maternal problem reported } \\
\text { and action taken }\end{array}$ & $\begin{array}{l}\text { Maternal problem reported } \\
\text { and action taken }\end{array}$ \\
\hline & Unadjusted & Adjusted & Unadjusted & Adjusted \\
\hline Estimation Type & Logistic & Logistic & Logistic & Logistic \\
\hline \multirow[t]{2}{*}{ CHW Call } & 0.47 & 0.23 & 0.73 & 0.50 \\
\hline & $(0.071-3.08)$ & $(0.021-2.58)$ & $(0.095-5.69)$ & $(0.047-5.39)$ \\
\hline \multirow[t]{2}{*}{ CHW Home Visit } & $3.50^{*}$ & $5.58^{*}$ & 1.38 & 1.69 \\
\hline & $(0.81-15.1)$ & $(0.88-35.4)$ & $(0.21-9.12)$ & $(0.18-15.5)$ \\
\hline Mean of standard of care arm & 0.13 & 0.13 & 0.83 & 0.83 \\
\hline $\mathrm{p}$-value for test of call = visit & 0.017 & 0.004 & 0.512 & 0.371 \\
\hline $\begin{array}{l}\mathrm{p} \text {-value for test of joint } \\
\text { significance of call and visit }\end{array}$ & 0.032 & 0.009 & 0.805 & 0.670 \\
\hline Controls? & N & Y & N & Y \\
\hline Observations & 83 & 79 & 83 & 53 \\
\hline \multicolumn{5}{|c|}{$\begin{array}{l}\text { Panel B. Newborn health problem } \\
\text { reporting and responses }\end{array}$} \\
\hline & (1) & (2) & (3) & (4) \\
\hline & $\begin{array}{l}\text { Any newborn health } \\
\text { problem reported }\end{array}$ & $\begin{array}{l}\text { Any newborn health } \\
\text { problem reported }\end{array}$ & $\begin{array}{l}\text { Newborn problem reported } \\
\text { and action taken }\end{array}$ & $\begin{array}{l}\text { Newborn problem reported } \\
\text { and action taken }\end{array}$ \\
\hline & Unadjusted & Adjusted & Unadjusted & Adjusted \\
\hline Estimation Type & Logistic & Logistic & Logistic & Logistic \\
\hline \multirow[t]{2}{*}{ CHW Call } & 1.27 & 1.21 & 1.30 & 0.95 \\
\hline & $(0.35-4.54)$ & $(0.33-4.46)$ & $(0.28-6.11)$ & $(0.20-4.57)$ \\
\hline \multirow[t]{2}{*}{ CHW Home Visit } & $3.53^{* *}$ & $3.78^{*}$ & 2.95 & $3.94^{*}$ \\
\hline & $(1.01-12.3)$ & $(0.95-15.1)$ & $(0.68-12.9)$ & $(0.80-19.4)$ \\
\hline Mean of standard of care arm & 0.21 & 0.21 & 0.13 & 0.13 \\
\hline$p$-value for test of call $=$ visit & 0.070 & 0.087 & 0.205 & 0.053 \\
\hline $\begin{array}{l}p \text {-value for test of joint } \\
\text { significance of call and visit }\end{array}$ & 0.078 & 0.120 & 0.260 & 0.104 \\
\hline Controls? & N & Y & N & Y \\
\hline Observations & 83 & 79 & 83 & 79 \\
\hline
\end{tabular}

*** $p<0.01,{ }^{* *} p<0.05, * p<0.1$

Notes: Odds ratios generated via logistic regression (models 1-4) are presented with $95 \%$ confidence intervals constructed with robust standard errors. "Any maternal problem reported" is a binary variable indicating whether or not the participant reported any maternal-related health problems at 10 days postnatal. "Maternal problem reported and action taken" is a binary variable taking the value 1 if the respondent reported both any maternal health problem or concern and taking any action to address the problem, including calling a health facility or pharmacy, visiting a facility or pharmacy, or another action. "Any infant problem reported" is a binary variable indicating whether or not the participant reported any infant-related health problems at 10 days postnatal. "Newborn problem reported and action taken" is a binary variable taking the value 1 if the respondent reported both any newborn health problem or concern and taking any action to address the problem, including calling a health facility or pharmacy, visiting a facility or pharmacy, or another action. For all adjusted regressions (columns 2 and 4), individual level covariates include the female participant's age (coded as an ordinal variable with values 18-25, 26-30, 31-35, or 36-40 years old), marital status (defined as a binary single or married), a binary variable indicating whether the female respondent was employed at any time in the past 12 months, a binary variable indicating whether the participant was enrolled in a concurrent randomized trial and received a voucher (cost subsidy) for postnatal family planning services, and a binary variable indicating whether the participant attended one or more antenatal care visits at a Jacaranda Health facility

practices assessed at 10 days and 9 weeks postdelivery, respectively (unadjusted model in column 3; adjusted model in column 4). We find an average knowledge in the standard of care arm of 3.2 items out of the maximum 6. While estimates of program impact of phone call assignment are positive in the unadjusted and adjusted models the differences from the standard of care group are not statistically significant.

Postnatal health practices at ten days and nine weeks are also assessed using a summative index. While estimates of a treatment effect are positive in both crude and adjusted models, they are substantively small and not statistically significant. 

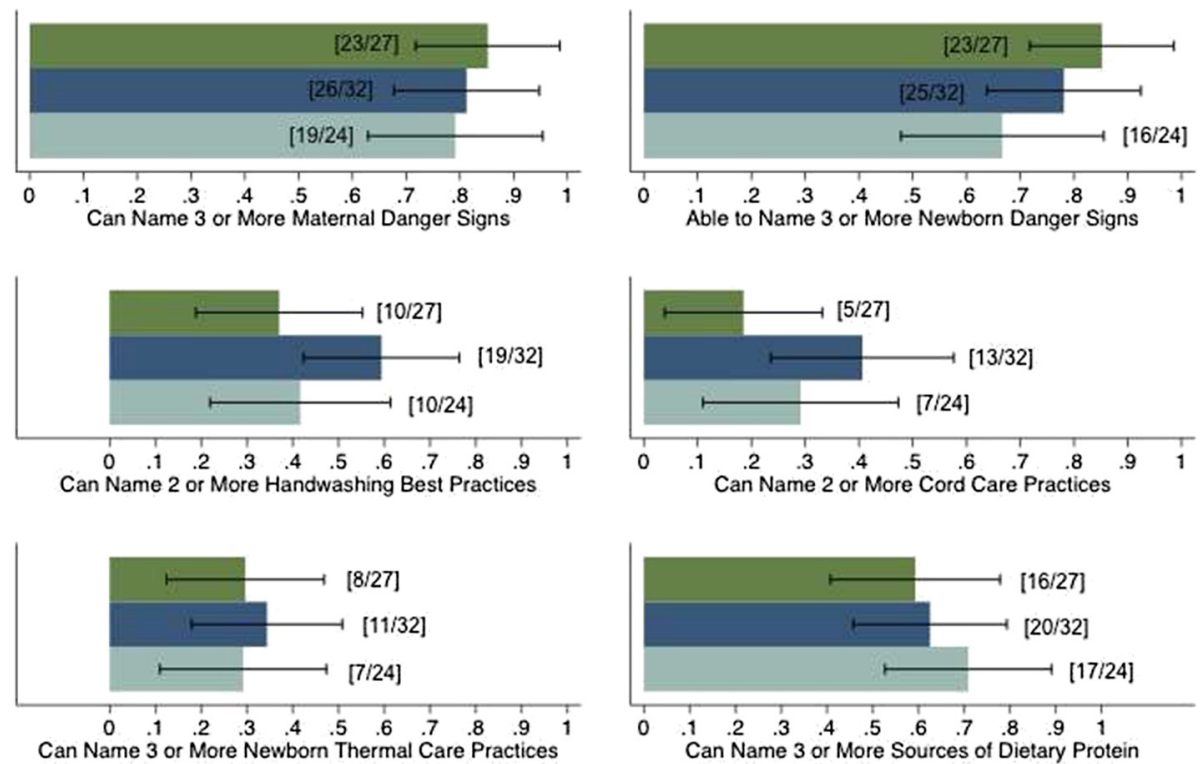

Standard of Care

CHW Phone Call

CHW Home Visit

Fig. 2 Self-reported knowledge of postnatal danger signs and health practices at day 10

\section{Limitations}

Our findings are subject to some important limitations. The sample size for this intervention was quite small. Our study was primarily powered to detect large effects of the intervention and thus our power to detect moderate effects was limited and most of the findings are not statistically significant at the $5 \%$ level. Furthermore, our study was not designed to be powered to detect changes in health outcomes. A substantially larger trial would be needed to determine the effect of similar interventions on key health outcomes such as mortality.

Further, this intervention took place in an urban setting and within partnerships at a social enterprise providing private low-cost maternal and newborn health care. Further research is necessary to evaluate if the findings seen could be replicated within the public sector, which has a larger geographical scale but also faces other challenges. Because our intervention relies on facilities to identify women who have recently delivered, it would not be suitable for providing postnatal care to women who deliver at home. Therefore this implementation strategy for delivering postnatal care is likely most relevant for urban areas such as Nairobi where rates of home birth are low (11.6\%) [5].

Furthermore, because Jacaranda Health is a private maternity facility, the patient profile may not represent women who deliver at home or in public facilities. Comparing the socioeconomic indicators of women in our sample to characteristics of the national urban sample in Kenya we find that our sample is similar in terms of access to an improved water source, with access to an improved water source for $90.8 \%$ of urban households in Kenya and $93 \%$ (71/ 76) of households in our sample [4]. Our sample has higher rates of access to improved non-shared toilet facilities at $(41 \%)$ compared to the national urban average of $30 \%$ [4].

Postnatal knowledge outcomes indicate that health knowledge was uniformly and unexpectedly high in our sample. In the context of relatively high overall knowledge, our estimate of the impact of the checklist interventions do not point to impacts of the intervention on knowledge. Replication of this evaluation may elucidate the potential knowledge gains of such an intervention in a less informed sample. Finally, while we were able to observe health seeking behavior through administrative data shared by our partner, many of the health practices measured in the study (such as breastfeeding and cord care practices) are self-reported. Some of the high reported rates of beneficial practices such as dry cord care and exclusive breastfeeding may be due to reporting bias.

Our study also had significant attrition, both in the implementation of the interventions and tracking of respondents at the ten-day follow-up survey and nine week follow-up survey. Estimates presented in Additional files 2, 3, 4, 5, 6 focus on a subsample that could be reached for the day 3 interventions. While the checklist delivered over the mobile phone was able to reach a larger share of respondents than 
a. Self-Reported Postpartum Practices at 10 Days Postpartum
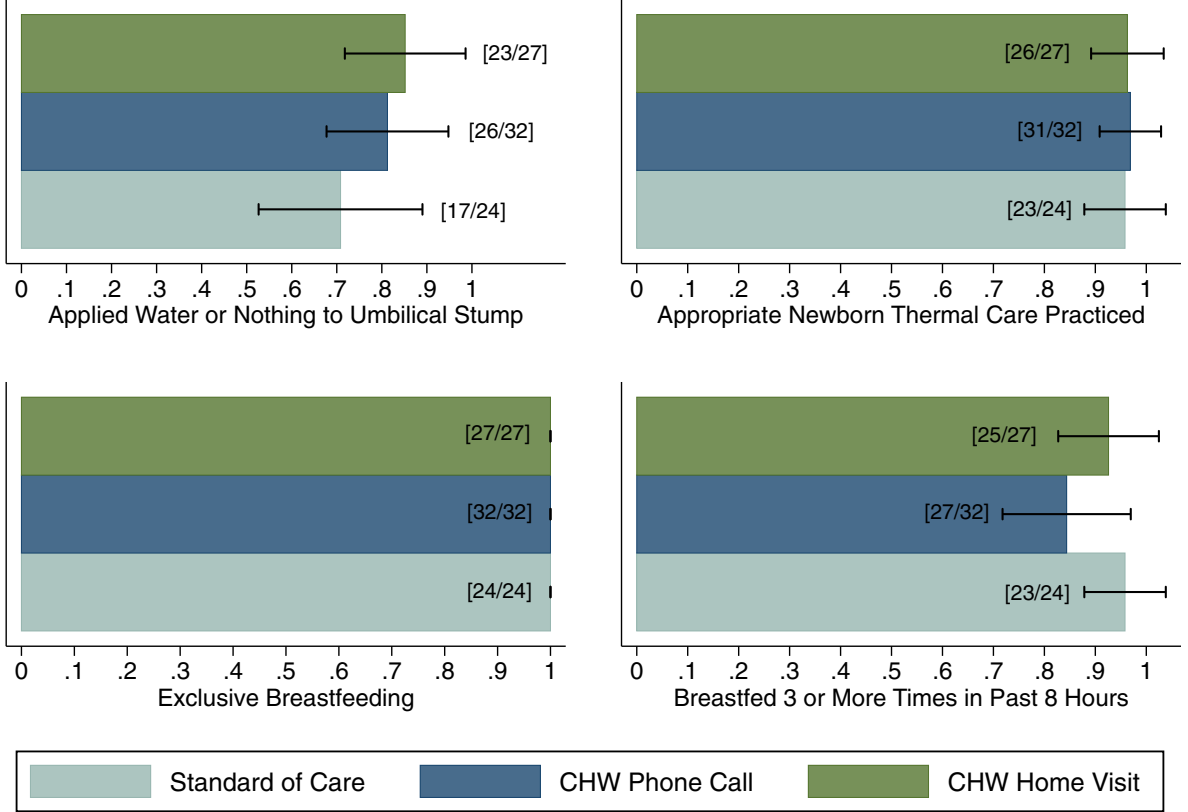

b. Self-Reported Postpartum Practices at 9 Weeks Postpartum
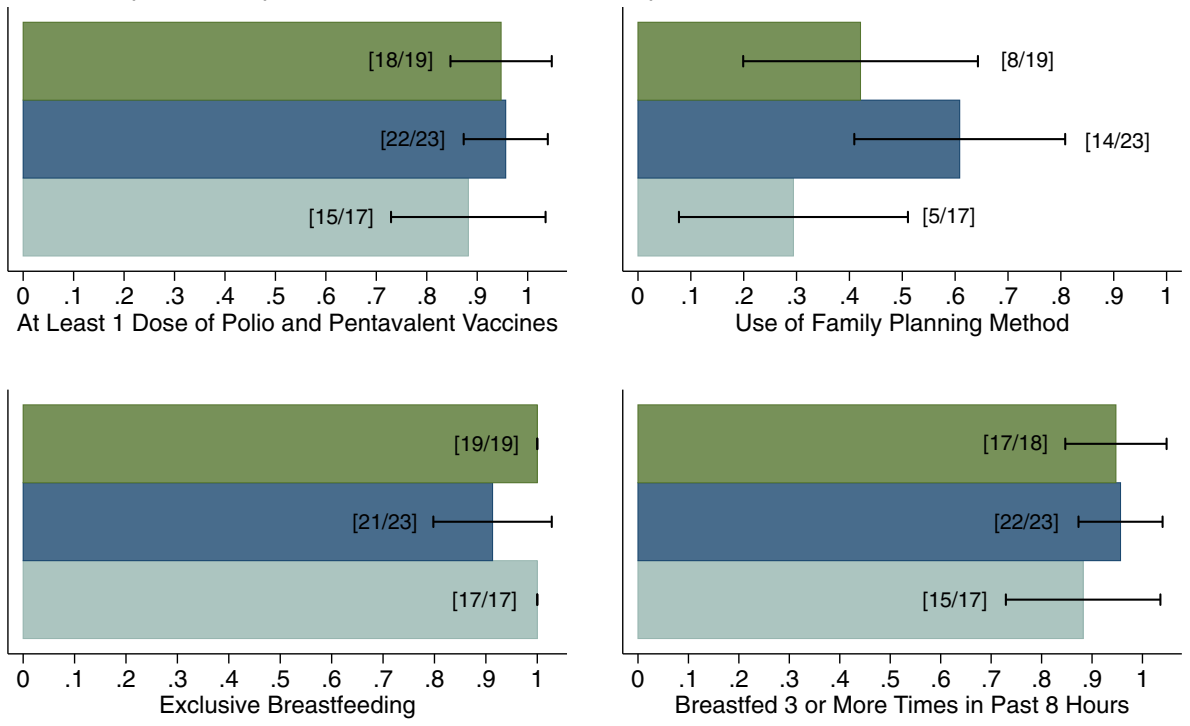

\section{Standard of Care}

CHW Phone Call

CHW Home Visit

Fig. 3 Self-reported postnatal health practices at 10 days and 9 weeks after delivery

the checklist administered in person, substantive conclusions are largely similar in both samples.

\section{Discussion}

Our findings suggest that CHWs can deliver an algorithmic, checklist-based postnatal health intervention either by phone or in a visit to women's homes three days after they give birth. Overall, the checklist was administered for $68 \%(50 / 73)$ of women who gave birth within three days of their delivery with more women reached over the phone than in person. Our study provides novel evidence of the feasibility of using checklists to support the work of CHWs in administering postnatal care in person and over mobile phones. While previous interventions to improve outcomes for mother and baby during the postpartum period have been complex and multifaceted, potentially involving as many as 11 visits over the entire period of a pregnancy $[7,8,13,24,25]$, our intervention 
Table 6 Self-reported postnatal health knowledge and practices

\begin{tabular}{lllll}
\hline & $(1)$ & $(2)$ & $(3)$ & $(4)$ \\
& $\begin{array}{l}\text { Index of postnatal health } \\
\text { knowledge, as reported at } \\
\text { day 10 post-delivery } \\
\text { Unadjusted }\end{array}$ & $\begin{array}{l}\text { Index of postnatal health } \\
\text { knowledge, as reported at } \\
\text { day 10 post-delivery } \\
\text { Adjusted }\end{array}$ & $\begin{array}{l}\text { Index of postnatal health } \\
\text { practices, as reported at day } 10 \\
\text { and 9 weeks post-delivery }\end{array}$ & $\begin{array}{l}\text { Index of postnatal health } \\
\text { practices, as reported at day } 10 \\
\text { and } 9 \text { weeks post-delivery }\end{array}$ \\
Adjusted
\end{tabular}

*** $p<0.01,{ }^{* *} p<0.05, * 0<0.1$

Notes: Coefficients generated via OLS regression (models 1-4) are presented with $95 \%$ confidence intervals constructed with robust standard errors. Postnatal health knowledge (models 1 and 2) is assessed as an ordinal variable generated using self-reported knowledge collected during the day 10 postnatal survey; knowledge is assessed as a summative index with a maximum of 6 points and a minimum of 0 points, where each point represents knowledge of the following 6 postnatal health topics: 1) ability to name 3 or more maternal danger signs; 2 ) ability to name 3 or more infant danger signs; 3 ) ability to name 2 or more hand washing best practices; 4) ability to name 2 or more; 5 ) ability to name 3 or more recognized newborn thermal care practices; 6 ) and ability to name 3 or more sources of maternal dietary protein. Postnatal health practices (models 3 and 4) assessed as an ordinal variable generated using self-reported practices collected during the day 10 and 9 week postnatal surveys; health behaviors are assessed as a summative index with a maximum of 8 points and a minimum of 0 points, where 4 points represent 4 key health practices reported at 10 day (exclusive breastfeeding, breastfed 3 or more times in the past 8 hours, appropriate newborn thermal care practices, and water or nothing applied to umbilical cord stump) and 4 points represent 4 key health practices reported at 9 weeks (exclusive breastfeeding, breastfed 3 or more times in the past 8 hours, use of postnatal contraception, infant has received at least one dose of polio and pentavalent vaccines). For all adjusted regressions (columns 2 and 4), individual level covariates include the female participant's age (coded as an ordinal variable with values 18-25, 26-30, 31-35, or 36-40 years old), marital status (defined as a binary single or married), a binary variable indicating whether the female respondent was employed at any time in the past 12 months, a binary variable indicating whether the participant was enrolled in a concurrent randomized trial and received a voucher (cost subsidy) for postnatal family planning services, and a binary variable indicating whether the participant attended one or more antenatal care visits at a Jacaranda Health facility

focused narrowly on postnatal care, piloting a mobile phone based version of a postnatal checklist that can be administered across a variety of health systems under different resource constraints. One advantage of this simple stand-alone approach is it addresses the concern that the wide scope of comprehensive home visit interventions presents a challenge to consistent distribution or intervention coverage to women and their newborns within existing community delivery systems [25].

Our intervention innovates by comparing the effectiveness of delivering a postnatal checklist entirely in person during a home visit or over mobile phones. Tools that leverage mHealth technology pose an opportunity to supplement existing community delivery systems, like CHW home visits, by increasing accessibility and coverage [26-28]. CHW home visit interventions in South Africa used mobile phone technology for data collection, patient registry, and health worker performance management, but not as the main point of contact for the intervention [8]. In urban areas, such as the ones we focus on in our study, mobile phone use is widespread and represents an opportunity for delivery of interventions that reach women in their homes.

Our study provides specific evidence on how postnatal checks can influence women's likelihood of seeking care and lead to earlier care-seeking in the crucial window immediately after birth. Overall, while some of our impact estimates are imprecise, the direction and magnitude of our findings suggest that the home visit administration of the checklist was especially helpful in encouraging timely careseeking in the critical postnatal window. The home visit increases the salience of infant health complications or concerns, with women assigned to the home visit treatment arm significantly more likely to report infant health problems at ten days after giving birth compared to the standard of care arm and more likely to both recognize and take action on complications detected in their newborns. 
The evidence that providing assistance and support to women in this window of time, which is both critical for their health and their newborns health but also filled with many other responsibilities and stresses, can increase timely care-seeking is consistent with evidence from Warren et al. (2015) that vouchers providing reimbursement for reproductive health services in Kenya can increase the number of newborns seen by facilities within their first 48 hours [29]. The significance of reducing delays in care-seeking is particularly important in the context of the peri-urban poor, where delays in care-seeking are common [30]. Such earlier linkages to facility-based care could have health implications, particularly as the first week of life poses significant risks. These risks are particularly important because the rapid progression of many neonatal illnesses, such as sepsis, pose notable risks to neonatal survival [31].

Our results highlight the limited care seeking behavior in the postpartum period for maternal health specifically. While newborns were brought to a health facility for care within 10 days of delivery $96 \%$ of the time in the standard of care arm (in 23 out of 24 cases), mothers receive care in a facility only $8 \%$ of the time (in 2 out of 24 cases). We see suggestive evidence that our interventions increased the likelihood of reporting a maternal complication and reduced delays in seeking care for maternal complications compared to the standard of care, though these differences are not statistically significant at conventional levels. Overall knowledge of both maternal and newborn danger signs is high across both standard of care and intervention arms in our sample, which suggests that addressing complications in both the immediate postnatal period and beyond would require increasing the likelihood of acting on perceived maternal health complications.

Across all three arms, we see high levels of knowledge and rates of beneficial postnatal practices such as breastfeeding, cord-care and vaccinations in our sample, resulting in very few statistically significant differences across the intervention treatment arms. We also see few substantive or statistically significant differences in practices and knowledge outcomes at the longer-term follow-up at 9 weeks across treatment arms, though we do see some suggestive but not statistically significant evidence of increases in the likelihood of using modern contraceptives. This evidence is consistent with findings from Watt et al. (2015) that one of the primary ways that the Safe Motherhood Voucher program in Kenya improved postnatal care was to improve counseling about family planning [32].

\section{Conclusions}

We find that a simple checklist, administered by CHWs over mobile phones or in person three days after giving birth, leads to earlier utilization of postnatal care and better recognition of potential mother and baby complications. Our evidence innovates by comparing delivery models for a targeted postnatal intervention that could be widely and affordably scaled.

\section{Endnotes}

${ }^{1}$ In order to avoid the possibility that patients responded to the phone call with more health seeking behaviors because of reciprocity, we include a phone call for the standard of care group that focused exclusively on customer service. Patients reported appreciating receiving any phone call so, to the extent that this call may have influenced behavior in the standard of care group, some estimates of program impacts may be underestimates.

${ }^{2}$ While our assessment guidelines are consistent with the WHO guidelines from 2014, our postnatal checklist was developed in 2013, prior to the release of these guidelines and was therefore not specifically based on these guidelines.

${ }^{3}$ Referrals were made to the nearest facility (not to Jacaranda) since Jacaranda was not offering emergency comprehensive pediatric care at the time of the intervention.

${ }^{4}$ We find highest uptake of the hormonal implant in both the standard of care and home visit arms (comprising 60\% $(3 / 5)$ and $50 \%(4 / 8)$ of participants reporting contraceptive use assigned to these arms, respectively). In the phoneadministered checklist arm, the most common method was oral contraceptives (comprising $50 \%$ of participants in this arm). In both the phone- and home visit-administered checklist arms, injectables were the second most common method (comprising $29 \%(4 / 14)$ and $25 \%$ (2/8) of participants reporting contraceptive use in these arms, respectively).

\section{Additional files}

Additional file 1: Postpartum screening checklist. (DOCX 1253 kb)

Additional file 2: Postpartum care-seeking behaviors, health problems and responses, and postpartum knowledge and health behaviors, among subsample of participants reached by the day 3 postpartum interventions. (DOCX $110 \mathrm{~kb}$ )

Additional file 3: Participant flow for subsample of participants reached by the day 3 postpartum interventions. (JPG 207 kb)

Additional file 4: Complications detected, referred, and referrals acted upon, among subsample of participants reached by the day 3 postpartum interventions. (JPG 45 kb)

Additional file 5: Self-reported knowledge of postnatal danger signs and health practices at day 10, among subsample of participants reached by the day 3 postpartum interventions. (JPG $80 \mathrm{~kb}$ )

Additional file 6: Self-reported postnatal health practices at 10 days and 9 weeks after delivery, among subsample of participants reached by the day 3 postpartum interventions. (PDF $61 \mathrm{~kb}$ )

Abbreviation

CHW, Community Health Worker 


\section{Acknowledgments}

Many thanks go to Nick Pearson and the staff of Jacaranda Health who supported this research from conception to implementation. We thank Grand Challenges Canada Saving Lives at Birth for their generous funding support of this research.

\section{Funding}

This research was supported by Grand Challenges Canada, funding award number 0166-03.

\section{Availability of data and materials}

De-identified data is available from the authors upon request.

\section{Authors' contributions}

$\mathrm{MM}$ and $\mathrm{JC}$ conceived of the project. MM, $\mathrm{AE}$ and $\mathrm{JC}$ designed the project. $A E$ and FM implemented the project in the field. MM, AE, CR and JC contributed to the statistical analysis. All authors were involved in drafting and revising the manuscript and approving the final version.

\section{Competing interests}

The authors declare they have no competing interests.

\section{Consent to publish}

Not applicable.

\section{Ethics and consent to participate}

This study was approved by Institutional Review Boards at Harvard School of Public Health (protocol \# IRB13-2714) and the Ethical and Scientific Review Committee of African Medical Research Foundation (AMREF) in Nairobi, Kenya (protocol 3 AMREF-ESRC P92/2013). All study participants provided written informed consent.

\section{Author details}

${ }^{1}$ Harvard T. H. Chan School of Public Health, Building 1, Room 1217, 665 Huntington Ave, Boston, MA 02115, USA. ${ }^{2}$ Jacaranda Health, P.O. Box 42844 - 00100, Nairobi, Kenya.

Received: 1 July 2015 Accepted: 24 May 2016

Published online: 04 June 2016

\section{References}

1. Requejo JH, Bryce J, Barros AJD, Berman P, Bhutta Z, Chopra M, et al. Countdown to 2015 and beyond: fulfilling the health agenda for women and children. Lancet. 2014; doi: 10.1016/S0140-6736(14)60925-9.

2. Kerber KJ, de Graft-Johnson JE, Bhutta ZA, Okong P, Starrs A, Lawn JE. Continuum of care for maternal, newborn, and child health: from slogan to service delivery. Lancet. 2007;370:1358-69.

3. World Health Organization. WHO Recommendations on Postnatal Care of the Mother and Newborn. World Health Organization. 2014. http:// apps.who.int/iris/bitstream/10665/97603/1/9789241506649_eng.pdf. Accessed 13 Jan 2015.

4. Kenya National Bureau of Statistics (KNBS) and ICF Macro. (2010) Kenya Demographic and Health Survey 2008-09. KNBS and ICF Macro. 2010. http://dhsprogram.com/pubs/pdf/fr229/fr229.pdf. Accessed 9 Jan 2015

5. Kenya National Bureau of Statistics (KNBS) and ICF Macro. Kenya Demographic and Health Survey: Key Indicators 2014. Rockville, MD: KNBS and ICF Macro; 2015

6. Beguy D, Elung'ata P, Mberu B, Oduor C, Wamukoya M, Nganyi B, Ezeh A. HDSS Profile: The Nairobi Urban Health and Demographic Surveillance System (NUHDSS). Int J Epidemiol. 2015; doi:10.1093/ije/dyu25.

7. Nalwadda CK, Waiswa P, Kiguli J, Namazzi G, Namutamba S, et al. High Compliance with Newborn Community-to-Facility Referral in Eastern Uganda: An Opportunity to Improve Newborn Survival. PLoS ONE. 2013; doi:10.1371/journal.pone.0081610.

8. Rotheram-Borus MJ, Tomlinson M, le Roux IM, Harwood JM, Comulada S, et al. A Cluster Randomised Controlled Effectiveness Trial Evaluating Perinatal Home Visiting among South African Mothers/Infants. PLoS One. 2014; doi:10.1371/journal.pone.0105934.

9. Gogia S, Sachdev HS. Home visits by CHWs to prevent neonatal deaths in developing countries: a systematic review. Bull World Health Organ. 2010;88:658-6B
10. Baqui AH, Ahmed S, El Arfeen S, Darmstdat G, Rosecrans A, Mannan I, Shman SM, Begum N, Mahmud AB, Seraji HR, Williams EK, Winch PJ, Santosham M, Black RE. Effect of timing of first postnatal care home visit on neonatal mortality on Bangladesh: a observational cohort study. BMJ. 2009;339:2826.

11. Zimba E, Kinney MV, Kachale F, Watensperger KZ, Blencowe $H$, et al. Newborn survival in Malawi: a decade of change and future implications. Health Policy Plann. 2012;27 Suppl 3:iii88-103.

12. World Health Organization and UNICEF. Home visits for the newborn child: a strategy to improve survival. World Health Organization. 2009. http:// whqlibdoc.who.int/hq/2009/WHO_FCH_CAH_09.02_eng.pdf. Accessed 12 Jan 2015.

13. Kirkwood BR, Manu A, ten Asbroek AH, Soremekun S, Weobong B, Gyan T, Danso S, Amenga-Etego S, Tawiah-Agyemang C, Owusu-Agyei S, Hill Z. Effect of the Newhints home-visits intervention on neonatal mortality rate and care practices in Ghana: a cluster randomized controlled trial. Lancet. 2013; doi:10.1016/S0140-6736(13)60095-1

14. Haider R, Ashworth A, Kabir I, Huttly SRA. Effect of community-based peer counselors on exclusive breastfeeding practices in Dhaka, Bangladesh: a randomized controlled trail. Lancet. 2000;356:1643-47.

15. Penfold S, Manzi F, Mkumbo E, Temu S, Jaribu J, Shamba DD, et al. Effect of homebased counseling on newborn care practices in southern Tanzania one year after implementation: a cluster-randomised controlled trial. BMC Pediatrics. 2014;14:187.

16. Duysburgh E, Kerstens B, Kouanda S, Kabore CP, Belemsaga Yugbara D, et al. Opportunities to improve postnatal care for mothers and infants: design of context-specific packages of postnatal interventions in rural districts in four sub-Saharan African countries. BMC Pregnancy Childbirth 2015; doi:10.1186/s12884-015-0562-8

17. Yonemoto N, Dowswell T, Nagai S, Mori R. Schedules for home visits in the early postnatal period. Cochrane Database of Systematic Reviews. 2013; doi:10.1002/14651858.CD009326.pub2

18. Kenya National Bureau of Statistics (KNBS). The 2009 Population and Housing Census Nairobi, Kenya. Calverton, Maryland: KNBS and ICF Macro; 2010.

19. Oliver M, Geniets A, Winters N, Rega I, Mbae S. What do community health workers have to say about their work and how can this inform improved programme design? A case studies with $\mathrm{CHWs}$ within Kenya. Global Health Action. 2015; doi:10.3402/gha.v8.27168.

20. World Health Organization and UNICEF. Caring for the Newborn at Home: A training course for CHWs: Community Health Worker Manual. World Health Organization. 2012. http://www.who.int/maternal_child_adolescent/news/ events/2012/CHW_Manual.pdf. Accessed 12 Jan 2015.

21. Buffington ST, Sibley LM, Beck DR, Armbruster DA. Home Based Life Saving Skills. American College of Nurse-Midwives. 2010. http://www.midwife.org/ ACNM/files/ccLibraryFiles/Filename/000000000548/TAC\%20Booklet_ print\%20ready.pdf. Accessed 19 Dec 2014.

22. O'Brien P. Procedures for comparing samples with multiple endpoints. Biometrics. 1984;40:1079-87.

23. Kling JR, Liebman JB, Katz LF. Experimental analysis of neighborhood effects Econometrica. 2007;75:83-119.

24. Darmstadt GL, Choi Y, Arifeen SE, Bari S, Rahman SM, Mannan I, et al. Evaluation of a Cluster-Randomized Controlled Trial of a Package of Community-Based Maternal and Newborn Interventions in Mirzapur, Bangladesh. PLoS ONE 2010; doi:10.1371/journal.pone.0009696.

25. Sitrin D, Guenther T, Murray J, Pilgrim N, Rubayet S, et al. Reaching Mothers and Babies with Early Postnatal Home Visits: The Implementation Realities of Achieving High Coverage in Large-Scale Programs. PLOS ONE. 2013; doi:10.1371/ journal.pone.0068930

26. Braun R, Catalani C, Wimbush J, Israelski D. CHWs and Mobile Technology: A Systematic Review of the Literature. PLOS ONE. 2013; doi:10.1371/journal. pone.0065772

27. Mehl $G$, Labrique A. Prioritizing integrated mHealth strategies for universal health coverage. Science. 2014;345:1284.

28. Mitchell M, Hedt B, Msellemu D, Mkaka M, Lesh N. Improvement in Integrated Management of Childhood IIIness (IMCI) Implementation through use of Mobile Technology: Evidence from a Pilot Study in Tanzania. BMC Med Inform Decis Mak. 2013;13:95.

29. Warren C, Abuya T, Kanya L, Obare F, Njuki R, Tennerman M. A cross sectional comparison of postnatal care quality in facilities participating in a maternal health voucher program versus non-voucher facilities in Kenya. BMC Pregnancy Childbirth. 2015;15:153.

30. Essendi H, Mills S, Fotso JC. Barriers to Formal Emergency Obstetric Care Services' Utilization. J Urban Health. 2011;88:356-369. 
31. Lawn JE, Cousens S, Zupan J. Neonatal Survival 1: 4 million neonatal deaths: When? Where? Why? Lancet. 2005; doi:10.1016/S0140-6736(05)71048-5.

32. Watt C, Abuya T, Warren C, Kanya, L., Bellows, B. Can reproductive health voucher programs improve quality of postnatal care? A quasi-experimental evaluation of Kenya's Safe Motherhood voucher scheme. PLoS ONE. 2015;10(4):e0122828.

33. Zupan J, Garner P, Omari AAA. Topical umbilical cord care at birth. Cochrane Database of Systematic Reviews. 2004; doi:10.1002/14651858. CD001057.pub2.

34. Capurro H. Topical umbilical cord care at birth. In: The WHO Reproductive Health Library. World Health Organization. 2004. http://apps.who.int/rhl/ newborn/cd001057_capurroh_com/en/. Accessed 19 Dec 2014.

Submit your next manuscript to BioMed Central and we will help you at every step:

- We accept pre-submission inquiries

- Our selector tool helps you to find the most relevant journal

- We provide round the clock customer support

- Convenient online submission

- Thorough peer review

- Inclusion in PubMed and all major indexing services

- Maximum visibility for your research

Submit your manuscript at www.biomedcentral.com/submit
Biomed Central 